\title{
A DEPENDENCE OF THE COST OF FAST CONTROLS FOR THE HEAT EQUATION ON THE SUPPORT OF INITIAL DATUM
}

\author{
HOAI-MINH NGUYEN
}

\begin{abstract}
The controllability cost for the heat equation as the control time $T$ goes to 0 is wellknown of the order $e^{C / T}$ for some positive constant $C$, depending on the controlled domain and for all initial datum. In this paper, we prove that the constant $C$ can be chosen to be arbitrarily small if the support of the initial data is sufficiently close to the controlled domain, but not necessary inside the controlled domain. The proof is in the spirit on Lebeau and Robbiano's approach in which a new spectral inequality is established. The main ingredient of the proof of the new spectral inequality is three-sphere inequalities with partial data.
\end{abstract}

Key words: heat equations, fast controls, controllability cost, spectral inequalities, three-sphere inequalities.

Mathematics Subject Classification: 93B05, 93B07, 93C20, 35A23, 35B30.

\section{INTRODUCTION}

We are interested in the dependence of the cost of fast controls for the heat equation on the support (location) of the initial data. Let $\omega \subsetneq \Omega$ be a bounded, open subset of $\mathbb{R}^{d}(d \geq 1), T>0$, $u_{0} \in L^{2}(\Omega)$, and $f \in L^{2}((0, T) \times \omega)$. Let $A$ be a Lipschitz, symmetric, uniformly elliptic, matrixvalued function defined in $\Omega$. Consider the unique solution $u \in L^{2}\left((0, T) ; H_{0}^{1}(\Omega)\right) \cap C\left([0, T] ; L^{2}(\Omega)\right)$ of the system

$$
\left\{\begin{array}{cl}
\partial_{t} u-\operatorname{div}(A(x) \nabla u)=f \mathbb{1}_{\omega} & \text { in }(0, T) \times \Omega, \\
u=0 & \text { on }(0, T) \times \partial \Omega, \\
u(t=0, \cdot)=u_{0} & \text { in } \Omega .
\end{array}\right.
$$

Here and in what follows, $\mathbb{1}_{D}$ denotes the characteristic function of a set $D$ of $\mathbb{R}^{d}$. It is well-known from the work of Gilles Lebeau and Luc Robbiano [8], via spectral inequalities and the work of Andrei Fursikov and Yu Imanuvilov [5], via Carleman's estimates that one can act on $\omega$ using $f$ to bring $u$ from the initial state $u_{0}$ (arbitrary) at time 0 to the final state 0 at time $T$ (arbitrarily positive).

For $D \subset \Omega$, set

$$
c(T, \omega, D)=\sup _{\substack{\left\|u_{0}\right\|_{L^{2}(\Omega)}=1 \\ \operatorname{supp} u_{0} \subset D}} \inf _{\substack{f \in L^{2}((0, T) \times \omega) \\ u(T, \cdot)=0 \text { where } u \text { satisfies }(1.1)}}\|f\|_{L^{2}((0, T) \times \omega) .} .
$$

For $T \in(0,1)$, one can prove that

$$
c_{1} e^{c_{2} / T} \leq c(T, \omega, \Omega) \leq C_{1} e^{C_{2} / T},
$$

for some positive constants $c_{1}, c_{2}, C_{1}$, and $C_{2}$ independent of $T$. The second inequality follows from the observability inequality [5,8], and the first inequality was obtained by Luc Miller [13] 
and others $[3,22]$. There is significant literature covering other aspects of the cost of the control for heat equations [2,4], the transport equation with small viscosities $[1,6,10,11]$, and the wave equation $[7,19,20]$. The cost of fast controls were also considered for linear thermoelasticity [9], Schrödinger equations $[4,11,12,18]$, and plate vibrations [12]. Similar questions were previously addressed in finite dimensions by Thomas Seidman [21].

The goal of this paper is to show a dependence of $c(T, \omega, D)$ on $D$. More precisely, we prove

Theorem 1.1. Let $T \in(0,1)$ and $\varepsilon>0$. Assume that $\omega \Subset \Omega$ is of class $C^{2}$, and set, for $r>0$,

$$
\omega_{r}=\left\{x \in \mathbb{R}^{d} ; \operatorname{dist}(x, \omega)<r\right\} \text {. }
$$

There exist two constants $\delta \in(0,1)$ and $C_{\varepsilon}>0$, depending only on $\varepsilon, \omega, \Omega$, and the elliptic and Lipschitz constants of $A$, such that

$$
c\left(T, \omega, \omega_{\delta}\right) \leq C_{\varepsilon} e^{\varepsilon / T} .
$$

Remark 1.1. The constants $\delta$ and $C_{\varepsilon}$ in Theorem 1.1 are independent of $T$.

When $\omega=\Omega$, the dependence of $c_{2}$ and $C_{2}$ on $\Omega$ has been studied extensively, see e.g. [3,13] and the references therein. Nevertheless, to our knowledge, the dependence of the cost on the support of initial datum for the heat equation has not been considered in the literature. Theorem 1.1 is new even in one dimensional case.

Theorem 1.1 is expected in the sense that if the support of the initial data is not too far from the controlled region, then it is easier to control. Even in this regard, this intuition is not completely transparent since the propagation speed is infinite and hence the support of the solution at any positive time is generally the whole domain $\Omega$. Known examples used in the moment method for the heat equations (mainly for one dimensional space) and other equations give the same size of the control cost for initial datum formed by eigenfunctions of the corresponding operator. From this aspect, Theorem 1.1 is thus unexpected.

The proof of Theorem 1.1 is in the spirit of Gilles Lebeau and Luc Robbiano's approach [8] in which we establish a new spectral inequality. Let $0<\lambda_{1} \leq \lambda_{2} \leq \ldots$ be the sequence of the eigenvalues of the operator $-\operatorname{div}(A \nabla \cdot)$ with the zero Dirichlet boundary condition, and let $e_{1}, e_{2}, \ldots$ be the corresponding eigenfunctions, i.e.,

$$
\left\{\begin{array}{cl}
-\operatorname{div}\left(A \nabla e_{i}\right)=\lambda_{i} e_{i} & \text { in } \Omega, \\
e_{i}=0 & \text { on } \partial \Omega .
\end{array}\right.
$$

Assume that $\left\{e_{i}, i \geq 1\right\}$ forms an orthogonal basis in $L^{2}(\Omega)$. Set, for $\lambda>0$,

$$
E_{\leq \lambda}=\left\{\sum_{\lambda_{i} \leq \lambda} a_{i} e_{i}(x) ; a_{i} \in \mathbb{R}\right\} .
$$

One of the key elements of Gilles Lebeau and Luc Robbiano's approach is the following spectral inequality

$$
\|v\|_{H^{1}(\Omega)} \leq C e^{C \sqrt{\lambda}}\|v\|_{L^{2}(\omega)} \quad \forall v \in E_{\lambda}
$$

where $C$ is a positive constant independent of $\lambda$.

In this paper, we also follow this approach. Nevertheless, to capture the dependence on the support of the initial datum, we use and establish the following new spectral inequality (compare with (1.8)). 
Proposition 1.1. Let $\varepsilon \in(0,1)$. There exist two constants $\delta \in(0,1)$ and $C_{\varepsilon}>0$, depending only on $\varepsilon, \omega, \Omega$, and the elliptic and Lipschitz constants of $A$, such that, for $\lambda>0$,

$$
\|v\|_{L^{2}\left(\omega_{\delta}\right)} \leq C_{\varepsilon} e^{\varepsilon \sqrt{\lambda}}\|v\|_{L^{2}(\omega)} \quad \forall v \in E_{\lambda} .
$$

Remark 1.2. It is important to emphasize here that the constants $\delta$ and $C_{\varepsilon}$ in Proposition 1.1 are independent of $\lambda$.

The proof of Proposition 1.1 is in the spirit of [8]. Nevertheless, we use three-sphere inequalities with partial data, which was recently established by the author, to quantitatively capture the dependence of the support. These inequalities have been derived and applied to the study of cloaking using negative-index materials $[15,16]$. A typical example of these inequalities is, see [16, Theorem 2.1],

Theorem 1.2. Let $d \geq 2, \Lambda \geq 1,0<R_{1}<R_{3}$, and let $\Gamma=\left\{x=\left(x^{\prime}, x_{d}\right) \in \partial B_{R_{1}} ; x_{d}=0\right\}$. Denote $O_{r}=\left\{x \in \mathbb{R}^{d} ; \operatorname{dist}(x, \Gamma)<r\right\}, D_{r}=B_{R_{3}} \backslash\left(\overline{B_{R_{1}} \cup O_{r}}\right)$, and $\Sigma_{r}=\partial B_{R_{1}} \backslash \bar{O}_{r}$ for $r>0$. For every $\alpha \in(0,1)$, there exists $r_{2} \in\left(0, R_{3}-R_{1}\right)$, depending only on $\alpha, \Lambda, \Gamma, R_{1}$, and $R_{3}$, such that for every $r_{1} \in\left(0, r_{2}\right)$, there exists $r_{0} \in\left(0, r_{1}\right)$, depending only on $r_{1}, \alpha, \Lambda, R_{1}$, and $R_{3}$, such that for $(d \times d)$ Lipschitz, uniformly elliptic, symmetric, matrix-valued function $\mathcal{M}$ defined in $D_{r_{0}}$ verifying, in $D_{r_{0}}$,

$$
\Lambda^{-1}|\xi|^{2} \leq\langle\mathcal{M}(x) \xi, \xi\rangle \leq \Lambda|\xi|^{2} \forall \xi \in \mathbb{R}^{d} \quad \text { and } \quad|\nabla \mathcal{M}(x)| \leq \Lambda,
$$

and for $V \in\left[H^{1}\left(D_{r_{0}}\right)\right]^{m}$ satisfying

$$
|\operatorname{div}(\mathcal{M} \nabla V)| \leq \Lambda_{1}(|\nabla V|+|V|) \text { in } D_{r_{0}} \text { for some } \Lambda_{1} \geq 0
$$

we have

$$
\|V\|_{H^{1}\left(B_{R_{1}+r_{2}} \backslash B_{R_{1}+r_{1}}\right)} \leq C\left(\|V\|_{H^{1 / 2}\left(\Sigma_{r_{0}}\right)}+\|\mathcal{M} \nabla V \cdot x /|x|\|_{H^{-1 / 2}\left(\Sigma_{r_{0}}\right)}\right)^{\alpha}\|V\|_{H^{1}\left(D_{r_{0}}\right)}^{1-\alpha},
$$

for some positive constant $C$, depending only on $\alpha, \Lambda, \Lambda_{1}, R_{1}, R_{3}$, and $d$.

The geometry of Theorem 1.2 is given in Figure 1.

We will use a variant of Theorem 1.2, given in Proposition 1.2, to derive Theorem 1.1. Nevertheless, we present Theorem 1.2 here to highlight the difference between the three-sphere inequalities used in this paper and the standard three-sphere ones. In (1.11), one only uses the information of $\Sigma_{r_{0}}$ (a portion of $\partial B_{R_{1}}$, see Figure 1) in the first interpolation term. The terminology partial data comes from this. The constants $r_{1}, r_{2}$, and $r_{0}$ are independent of $\Lambda_{1}$, but the constant $C$ does depend on $\Lambda_{1}$. If instead of $\Sigma_{r_{0}}$, one uses $\partial B_{R_{1}}$, inequality (1.11) is then known. Using known three-sphere inequalities and the arguments of the propagation of smallness, one can prove (1.2) for some $\alpha \in(0,1)$. Nevertheless, the non-triviality and the novelty of Theorem 1.2 rely on the fact that, for a given arbitrary $\alpha \in(0,1),(1.11)$ holds for some $r_{0}, r_{1}, r_{2}$. Even if $v$ is a solution of the Laplace equation in two dimensions, using Hadamard three-sphere (circles) inequalities and the arguments of propagation of smallness, as far as we know, one can only obtain (1.11) for some small $\alpha$, even though one replaces $\Sigma_{r_{0}}$ by $\partial B_{R_{1}} \backslash\left\{x_{0}\right\}$ for some $x_{0} \in \partial B_{R_{1}}$. The possibility to take $\alpha$ close to 1 is crucial for the proof of Theorem 1.1 where $\varepsilon$ can be arbitrarily small. This point is also crucial for the cloaking applications considered in [15,16]. Several applications of Theorem 1.2 concerning variants of Hadamard's three-circle inequalities with partial data are given in [16]. 


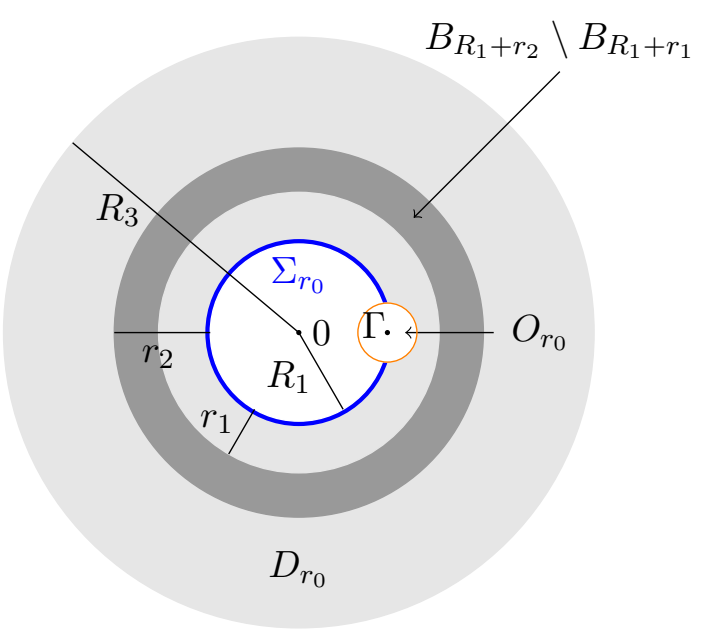

FiguRE 1. Geometry of Theorem 1.2 in two dimensions

We now introduce some notations to state the local version of Theorem 1.2, which is used in the proof of Proposition 1.1. For $d \geq 2$ and $x=\left(x_{1}, x_{2}, \widetilde{x}\right) \in \mathbb{R} \times \mathbb{R} \times \mathbb{R}^{d-2}$, we use the polar coordinate $(\hat{r}, \theta)$ with $\theta \in(-\pi, \pi]$ for the pair $\left(x_{1}, x_{2}\right)$; the variable $\widetilde{x}$ is irrelevant for $d=2$. For $0<\gamma_{1}<\gamma_{2}<1$ and for $R>0$, we denote

$$
Y_{\gamma_{1}, \gamma_{2}, R}=\left\{x \in \mathbb{R}^{d} ; \theta \in(-\pi / 2, \pi / 2), \gamma_{1} R<\hat{r}<\gamma_{2} R, \text { and }|\widetilde{x}|<R\right\},
$$

(see also Figure 2). The following variant of Theorem 1.2 in a half plane, see [16, Theorem 3.1], is the key ingredient of the proof of Proposition 1.1.

Proposition 1.2. Let $d \geq 2, \Lambda \geq 1$, and $R_{*}<R<R^{*}$. Then, for any $\alpha \in(0,1)$, there exists a constant $\hat{\gamma}_{2} \in(0,1)$, depending only on $\alpha, \Lambda, R_{*}, R^{*}$, and $d$ such that for every $\hat{\gamma}_{1} \in\left(0, \hat{\gamma}_{2}\right)$, there exists $\hat{\gamma}_{0} \in\left(0, \hat{\gamma}_{1}\right)$, depending only on $\alpha, \hat{\gamma}_{1}, \Lambda, R_{*}, R^{*}$, and $d$ such that, for real, symmetric, uniformly elliptic, Lipschitz matrix-valued functions $\mathcal{M}$ defined in $D_{\hat{\gamma}_{0}}:=Y_{\hat{\gamma}_{0}, 1, R}$ verifying, in $D_{\hat{\gamma}_{0}}$,

$$
\Lambda^{-1}|\xi|^{2} \leq\langle\mathcal{M}(x) \xi, \xi\rangle \leq \Lambda|\xi|^{2} \forall \xi \in \mathbb{R}^{d} \quad \text { and } \quad|\nabla \mathcal{M}(x)| \leq \Lambda,
$$

and for $V \in\left[H^{1}\left(D_{\hat{\gamma}_{0}}\right)\right]^{m}$ satisfying

$$
|\operatorname{div}(\mathcal{M} \nabla V)| \leq \Lambda_{1}(|\nabla V|+|V|) \text { in } D_{\hat{\gamma}_{0}} \text { for some } \Lambda_{1} \geq 0,
$$

we have, with $\Sigma_{\hat{\gamma}_{0}}=\partial D_{\hat{\gamma}_{0}} \cap\left\{x_{1}=0\right\}$,

$$
\|V\|_{H^{1}\left(Y_{\hat{\gamma}_{1}, \hat{\gamma}_{2}, \frac{R}{4}}\right)} \leq C\left(\|V\|_{H^{1 / 2}\left(\Sigma_{\hat{\gamma}_{0}}\right)}+\left\|\mathcal{M} \nabla V \cdot \eta_{1}\right\|_{H^{-1 / 2}\left(\Sigma_{\hat{\gamma}_{0}}\right)}\right)^{\alpha}\|V\|_{H^{1}\left(D_{\hat{\gamma}_{0}}\right)}^{1-\alpha},
$$

for some positive constant $C$, depending only on $\alpha, \hat{\gamma}_{1}, \Lambda, \Lambda_{1}, R_{*}, R^{*}$, and $d$.

Here and in what follows, $\eta_{1}, \cdots, \eta_{d}$ denotes the standard basis of $\mathbb{R}^{d}$, i.e., $\eta_{1}=(1,0, \ldots, 0)$, $\ldots, \eta_{d}=(0, \ldots, 0,1)$.

The proof of Proposition 1.2 given in [16] is quite delicate and involves new (uniform) Carleman's inequalities applied to second-order elliptic equations in which the coefficients might be degenerate and in which the geometry of the considered domain is taken into account in the 


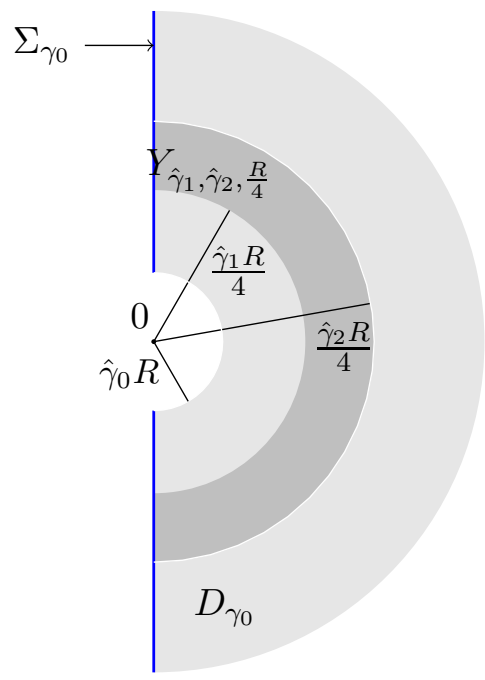

FiguRE 2. Geometry of $Y_{\gamma_{1}, \gamma_{2}, \frac{R}{4}}, \Sigma_{\gamma_{0}}$, and $D_{\gamma_{0}}$ in two dimensions.

proof. The proof is much simpler for the case $A=I$ and $d=2$, but already contains several key ideas [15].

The paper is organized as follows. Section 2 is devoted to the proof of Proposition 1.1. The proof of Theorem 1.1 is given in Section 3.

\section{Spectral inequality}

This section is devoted to the proof of Proposition 1.1. The key ingredient of the proof is:

Lemma 2.1. Let $M$ be a Lipschitz, symmetric, uniformly elliptic, matrix-valued defined in $\Omega \times$ $(-1,1)$ and let $\varphi \in H^{1}(\Omega \times(-1,1))$ be such that

$$
|\operatorname{div}(M \nabla \varphi)| \leq \Lambda(|\nabla \varphi|+|\varphi|) \text { in } \Omega \times(-1,1) .
$$

Set

$$
D_{r}=\left\{X=\left(x, x_{d+1}\right) \in \mathbb{R}^{d+1} ; \operatorname{dist}(X, \omega \times\{0\})<r\right\} \text { for } r>0 .
$$

Given $\alpha \in(0,1)$, there exist two constants $\delta \in(0,1)$ and $C_{\alpha}>0$, depending only on $\alpha, \omega, \Omega$, and the Lipschitz and elliptic constants of $M$, such that

$$
\|\varphi\|_{H^{1}\left(D_{\delta}\right)} \leq C_{\alpha}\left(\|\varphi\|_{H^{1 / 2}(\omega \times\{0\})}+\left\|M \nabla \varphi \cdot \eta_{d+1}\right\|_{H^{-1 / 2}(\omega \times\{0\})}\right)^{\alpha}\|\varphi\|_{H^{1}(\Omega \times(-1,1))}^{1-\alpha} .
$$

Remark 2.1. The constant $\delta$ and $C_{\alpha}$ are independent of $\varphi$.

Proof of Lemma 2.1. Since $\omega$ is of class $C^{2}$, by using local charts and a change of variables, it suffices to prove the following result: Let $\hat{M}$ be a Lipschitz, symmetric, uniformly elliptic, matrix-valued function defined in $Q:=(-1,1)^{d+1}$, and let $\hat{\varphi} \in H^{1}(Q)$ be such that

$$
|\operatorname{div}(\hat{M} \nabla \hat{\varphi})| \leq \hat{\Lambda}(|\nabla \hat{\varphi}|+|\hat{\varphi}|) \text { in } Q
$$




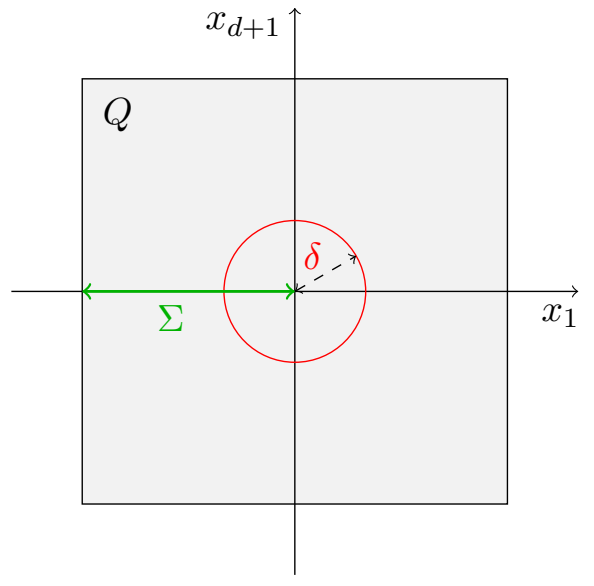

a)

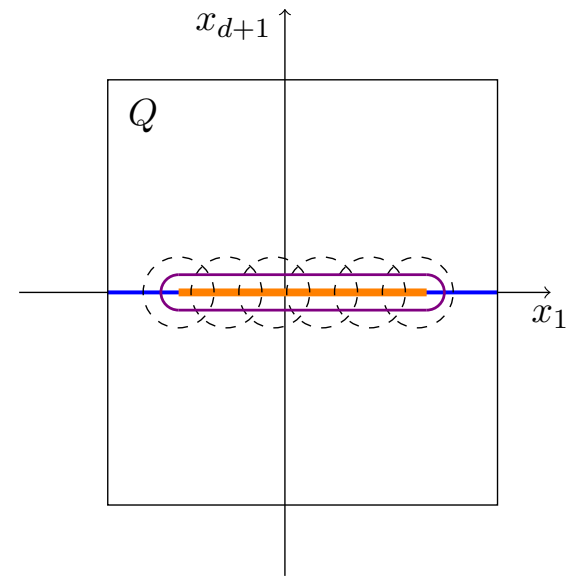

b)

Figure 3. a): Geometry of inequality 2.3 in two dimensions with $\Sigma:=Q \cap\left\{x_{d+1}=\right.$ $\left.\left.0 ; x_{1}<0\right\} . b\right)$ The way to obtain (2.1) from (2.3) for $d=1 ; \omega$ is the orange interval, $\Omega$ is the blue interval, $D_{\delta}$ is the region whose boundary is violet.

Given $\alpha \in(0,1)$, there exists $\delta>0$, depending only on $\alpha$, and the Lipschitz and elliptic constants of $\hat{M}$, such that

$$
\|\hat{\varphi}\|_{H^{1}\left(B_{\delta}\right)} \leq C\left(\|\hat{\varphi}\|_{H^{1 / 2}(\Sigma)}+\left\|\hat{M} \nabla \hat{\varphi} \cdot \eta_{d+1}\right\|_{H^{-1 / 2}(\Sigma)}\right)^{\alpha}\|\hat{\varphi}\|_{H^{1}(Q)}^{1-\alpha},
$$

where $\Sigma:=Q \cap\left\{x_{d+1}=0 ; x_{1}<0\right\}$ for some positive constant $C$ depending only on $\alpha$, the Lipschitz and elliptic constants of $\hat{M}$, and $\hat{\Lambda}$.

Here and in what follows in this proof, $B_{r}$ denotes the open ball centered at 0 and of radius $r>0$ in $\mathbb{R}^{d+1}$.

It is important to note that in (2.3), the norms in the RHS are considered in the set $\Sigma$ which is defined by $Q \cap\left\{x_{d+1}=0 ; x_{1}<0\right\}$ and is not given by the set $Q \cap\left\{x_{d+1}=0\right\}$. See a) of Figure 3 for the geometry of (2.3) and b) of Figure 3 for the ideas behind using local charts and covering arguments to obtain (2.1) from (2.3).

We will make a change of variables in order to apply Proposition 1.2. To this end, for $X=$ $\left(x_{1}, \cdots, x_{d+1}\right) \in Q \backslash\left\{x_{d+1}=0 ; x_{1} \leq 0\right\}$, define

$$
\mathcal{R}(X)=\left(y_{1}, x_{2}, \cdots, x_{d}, y_{d+1}\right)
$$

with $\left(y_{1}, y_{d+1}\right)=\hat{r} e^{i \theta / 2}$ if $\left(x_{1}, x_{d+1}\right)=\hat{r} e^{i \theta}$ for $\hat{r}>0$, and $\theta \in(-\pi, \pi)$.

Set

$$
\hat{\varphi}_{1}=\hat{\varphi} \circ \mathcal{R}^{-1} \text { in } \hat{Q}_{1}:=\mathcal{R}\left(Q \backslash\left\{x_{d+1}=0 ; x_{1} \leq 0\right\}\right) .
$$

Set

and

$$
f(x)=\operatorname{div}(\hat{M} \nabla \hat{\varphi})(x) \text { in } Q, \quad f_{1}(x)=\frac{f}{|\operatorname{det}(\nabla \mathcal{R})|} \circ \mathcal{R}^{-1}(x) \text { in } Q_{1},
$$

$$
\hat{M}_{1}=\frac{\nabla \mathcal{R} \hat{M} \nabla \mathcal{R}^{\top}}{|\operatorname{det}(\nabla \mathcal{R})|} \circ \mathcal{R}^{-1} \text { in } \hat{Q}_{1} .
$$


It is clear from (2.4) that the elliptic and Lipschitz constants of $\hat{M}_{1}$ are bounded by the elliptic and Lipschitz constants of $\hat{M}$, up to a constant $C$, depending only on $d$.

Since $\operatorname{div}(\hat{M} \nabla \hat{\varphi})=f$ in $Q$, it follows from a change of variables that

$$
\operatorname{div}\left(\hat{M}_{1} \nabla \hat{\varphi}_{1}\right)=f_{1} \text { in } Q_{1} .
$$

We have

$$
\begin{gathered}
\operatorname{det}(\nabla \mathcal{R})(x)=1 / 2 \text { for } x \in Q, \\
|\nabla \hat{\varphi}(x)| \leq|\nabla \mathcal{R}(x)|\left|\nabla \hat{\varphi}_{1} \circ \mathcal{R}(x)\right| \leq C\left|\nabla \hat{\varphi}_{1} \circ \mathcal{R}(x)\right| \text { for } x \in Q .
\end{gathered}
$$

Since $|f| \leq C(|\nabla \varphi|+|\varphi|)$ in $Q$ by (2.2), we derive from (2.5) that

$$
\left|\operatorname{div}\left(\hat{M}_{1} \nabla \hat{\varphi}_{1}\right)\right| \leq \hat{\Lambda}_{1}\left(\left|\nabla \varphi_{1}\right|+\left|\varphi_{1}\right|\right) \text { in } Q_{1},
$$

for some $\hat{\Lambda}_{1}>0$, depending only on $\Lambda$ and $d$.

Set

$$
\Gamma_{1,+}=\left\{\left(x_{1}, \ldots, x_{d+1}\right) ; x_{1}=0, x_{d+1}>0, x_{j} \in(-1,1) \text { for } 2 \leq j \leq d\right\}
$$

and

$$
\Gamma_{1,-}=\left\{\left(x_{1}, \ldots, x_{d+1}\right) ; x_{1}=0, x_{d+1}<0, x_{j} \in(-1,1) \text { for } 2 \leq j \leq d\right\} .
$$

Apply Proposition 1.2 to $\hat{\varphi}_{1}$ with $R=1, \hat{\gamma}_{1}=\hat{\gamma}_{2} / 2$, and $\hat{\gamma}_{0}=0$, and in $\mathbb{R}^{d+1}$ with $\left(x_{1}, x_{2}, \widetilde{x}\right)$ being replaced by $\left(x_{1}, x_{d+1},\left(x_{2}, \cdots, x_{d}\right)\right)$. There exists $\hat{\gamma}_{2}>0$ such that

$$
\begin{aligned}
& \left\|\hat{\varphi}_{1}\right\|_{H^{1}\left(\left(B_{\hat{\gamma}_{2}} \backslash B_{\hat{\gamma}_{2} / 2}\right) \cap\left\{x_{1}>0\right\}\right)} \leq C\left\|\hat{\varphi}_{1}\right\|_{H^{1}\left(\hat{Q}_{1}\right)}^{1-\alpha} \\
& \times\left(\left\|\hat{\varphi}_{1}\right\|_{H^{1 / 2}\left(\Gamma_{1,+}\right)}+\left\|\hat{M}_{1} \nabla \hat{\varphi}_{1} \cdot \eta_{1}\right\|_{H^{1 / 2}\left(\Gamma_{1,+}\right)}+\left\|\hat{\varphi}_{1}\right\|_{H^{1 / 2}\left(\Gamma_{1,-}\right)}+\left\|\hat{M}_{1} \nabla \hat{\varphi}_{1} \cdot \eta_{1}\right\|_{H^{1 / 2}\left(\Gamma_{1,-}\right)}\right)^{\alpha} .
\end{aligned}
$$

Since $\hat{\varphi}=\hat{\varphi}_{1} \circ \mathcal{R}$ in $Q$, it follows from a change of variables, see e.g. [14, Lemma 2], that

$$
\|\hat{\varphi}\|_{H^{1}\left(\left(B_{\hat{\gamma}_{2}} \backslash B_{\hat{\gamma}_{2} / 2}\right) \backslash\left\{x_{d+1}=0 ; x_{1}<0\right\}\right)} \leq C\|\hat{\varphi}\|_{H^{1}(Q)}^{1-\alpha}\left(\|\hat{\varphi}\|_{H^{1 / 2}(\Sigma)}+\left\|\hat{M} \nabla \hat{\varphi} \cdot \eta_{d+1}\right\|_{H^{-1 / 2}(\Sigma)}\right)^{\alpha} .
$$

Since $\hat{\varphi} \in H^{1}(Q)$, and hence in particular $\hat{\varphi} \in H^{1}\left(B_{\hat{\gamma}_{2}}\right)$, we obtain

$$
\|\hat{\varphi}\|_{H^{1}\left(B_{\hat{\gamma}_{2}} \backslash B_{\hat{\gamma}_{2} / 2}\right)} \leq C\|\hat{\varphi}\|_{H^{1}(Q)}^{1-\alpha}\left(\|\hat{\varphi}\|_{H^{1 / 2}(\Sigma)}+\left\|\hat{M} \nabla \hat{\varphi} \cdot \eta_{d+1}\right\|_{H^{-1 / 2}(\Sigma)}\right)^{\alpha} .
$$

Using the fact

$$
|\operatorname{div}(\hat{M} \nabla \hat{\varphi})| \leq \hat{\Lambda}(|\nabla \varphi|+|\varphi|) \text { in } Q
$$

and $\hat{M}$ is symmetric, uniformly elliptic and Lipschitz, one has ${ }^{1}$

$$
\|\hat{\varphi}\|_{H^{1}\left(B_{\gamma_{2}}\right)} \leq C\|\hat{\varphi}\|_{H^{1}\left(B_{\gamma_{2}} \backslash B_{\gamma_{2} / 2}\right)} .
$$

Assertion (2.3) now follows from (2.8) and (2.9) with $\delta=\gamma_{2}$. The proof is complete.

Remark 2.2. One of the key points of the proof is the assertion (2.3). This assertion is known if one replaces the set $Q \cap\left\{x_{d+1}=0 ; x_{1}<0\right\}$ by $Q \cap\left\{x_{d+1}=0\right\}$ and the proof in this case can be done as in [8]. However, this does not imply (2.1). The proof of (2.3) follows from Proposition 1.2, which is non-trivial.

We are ready to give

\footnotetext{
${ }^{1}$ One can prove (2.9) using a contradiction argument and the unique continuation principle.
} 
Proof of Proposition 1.1. Since $v \in E_{\leq \lambda}$, there exists $a_{i} \in \mathbb{R}$ with $\lambda_{i} \leq \lambda$, such that

$$
v(x)=\sum_{\lambda_{i} \leq \lambda} a_{i} e_{i}(x) \text { in } \Omega .
$$

As in the spirit of [8], set, with $X=\left(x, x_{d+1}\right) \in \Omega \times \mathbb{R}$,

$$
V(X)=\sum_{\lambda_{i} \leq \lambda} \lambda_{i}^{-1 / 2} a_{i} \sinh \left(\lambda_{i}^{1 / 2} x_{d+1}\right) e_{i}(x),
$$

where $\sinh t=\frac{1}{2}\left(e^{t}-e^{-t}\right)$ for $t \in \mathbb{R}$. Since $-\operatorname{div}_{x}\left(A(x) \nabla e_{i}(x)\right)=\lambda_{i} e_{i}(x)$ in $\Omega$, it follows that

$$
\left\{\begin{array}{c}
\partial_{x_{d+1}}^{2} V+\operatorname{div}_{x}\left(A(x) \nabla_{x} V\right)=0 \text { in } \Omega \times \mathbb{R}, \\
V(X)=0 \text { for } X \in \Omega \times\{0\}, \\
\partial_{x_{d+1}} V(X)=v(x) \text { for } X \in \Omega \times\{0\} .
\end{array}\right.
$$

Given $\alpha \in(0,1)$, by applying Lemma 2.1 to $V$, there exist two constants $\delta=\delta(\alpha) \in(0,1)$ and $C_{\alpha}>0$, depending only on $\alpha, \omega, \Omega$, and the elliptic and Lipschitz constants of $A$, such that

$$
\|V\|_{H^{1}\left(D_{2 \delta}\right)} \leq C_{\alpha}\left(\|V\|_{H^{1 / 2}(\omega \times\{0\})}+\left\|\partial_{x_{d+1}} V\right\|_{H^{-1 / 2}(\omega \times\{0\})}\right)^{\alpha}\|V\|_{H^{1}(\Omega \times(-1,1))}^{1-\alpha} .
$$

Using (2.10), we derive from (2.11) that

$$
\|V\|_{H^{1}\left(D_{2 \delta}\right)} \leq C_{\alpha}\|v\|_{L^{2}(\omega)}^{\alpha}\|V\|_{H^{1}(\Omega \times(-1,1))}^{1-\alpha} .
$$

Since $A$ is Lipschitz, by the regularity theory of elliptic equations ${ }^{2}$, one has

$$
\left\|\partial_{x_{d+1}} V\right\|_{L^{2}\left(\omega_{\delta}\right)} \leq C_{\alpha}\|V\|_{H^{1}\left(D_{2 \delta}\right)},
$$

which yields

$$
\|v\|_{L^{2}\left(\omega_{\delta}\right)} \leq C_{\alpha}\|V\|_{H^{1}\left(D_{2 \delta}\right)} .
$$

On the other hand, by the standard spectral inequality (1.8), one gets

$$
\|V\|_{H^{1}(\Omega \times(-1,1))} \leq C e^{C \sqrt{\lambda}}\|v\|_{L^{2}(\omega)},
$$

for some positive constant $C$, depending only on $\omega, \Omega$, and the elliptic and Lipschitz constants of A.

Combining (2.12), (2.13) and (2.14) yields

$$
\|v\|_{L^{2}\left(\omega_{\delta}\right)} \stackrel{(2.13)}{\leq} C_{\alpha}\|V\|_{H^{1}\left(D_{2 \delta}\right)} \stackrel{(2.12)}{\leq} C_{\alpha}\|v\|_{L^{2}(\omega)}^{\alpha}\|V\|_{H^{1}(\Omega \times(-1,1))}^{1-\alpha} \stackrel{(2.14)}{\leq} C_{\alpha} e^{C(1-\alpha) \sqrt{\lambda}}\|v\|_{L^{2}(\omega)} .
$$

By choosing $\alpha$ such that $C(1-\alpha)=\varepsilon$, we derive from (2.15) that

$$
\|v\|_{L^{2}\left(\omega_{\delta}\right)} \leq C_{\varepsilon} e^{\varepsilon \sqrt{\lambda}}\|v\|_{L^{2}\left(\omega_{\delta}\right)} .
$$

The proof is complete.

\footnotetext{
${ }^{2}$ One can directly apply the quotient method due to Louis Nirenberg [17].
} 


\section{Proof of Theorem 1.1}

The proof of Theorem 1.1 is based on the following lemma, which will be derived from the spectral inequality stated in Proposition 1.1.

Lemma 3.1. Let $0<T<1, \lambda>0$, and let $v_{0} \in E_{\lambda}$. Let $v \in L^{2}\left((0, T), H_{0}^{1}(\Omega)\right) \cap C\left([0, T], L^{2}(\Omega)\right)$ be the unique solution of the system

$$
\left\{\begin{array}{cl}
\partial_{t} v-\operatorname{div}(A \nabla v)=0 & \text { in }(0, T) \times \Omega, \\
v=0 & \text { on }(0, T) \times \partial \Omega, \\
v(t=0, \cdot)=v_{0} & \text { in } \Omega .
\end{array}\right.
$$

For $\varepsilon>0$, there exist two constants $\delta \in(0,1)$ and $C_{\varepsilon}>0$, depending only on $\varepsilon, \omega$, and $\Omega$, and the elliptic and Lipschitz constants of $A$, such that

$$
\|v(T, \cdot)\|_{L^{2}\left(\omega_{\delta}\right)} \leq C_{\varepsilon} \delta^{-1} T^{-1 / 2} e^{\varepsilon \sqrt{\lambda}}\|v\|_{L^{2}((0, T) \times \omega)} .
$$

Recall that $\omega_{r}$ is defined in (1.4).

Remark 3.1. The constants $\delta$ and $C_{\varepsilon}$ in Lemma 3.1 are independent of $\lambda$ and $T$.

Proof. By Proposition 1.1, there exist $\delta \in(0,1)$ and $C_{\varepsilon}>0$, such that

$$
\|\xi\|_{L^{2}\left(\omega_{2 \delta}\right)} \leq C_{\varepsilon} e^{\varepsilon \sqrt{\lambda}}\|\xi\|_{L^{2}(\omega)} \text { for } \xi \in E_{\leq \lambda} .
$$

Since $v_{0} \in E_{\leq \lambda}$, it follows that $v(t, \cdot) \in E_{\leq \lambda}$ for $t \in(0, T)$. We derive from (3.2) that

$$
\|v(t, \cdot)\|_{L^{2}\left(\omega_{2 \delta}\right)} \leq C_{\varepsilon} e^{\varepsilon \sqrt{\lambda}}\|v(t, \cdot)\|_{L^{2}(\omega)} \text { for } t \in(0, T) .
$$

Fix $\varphi \in C_{c}^{\infty}\left(\mathbb{R}^{d}\right)$ such that $0 \leq \varphi \leq 1, \varphi=1$ in $\omega_{\delta}$, $\operatorname{supp} \varphi \subset \omega_{2 \delta}$, and $\left|\nabla_{x}^{\alpha} \varphi\right| \leq C / \delta^{|\alpha|}$ for all multi-indices $\alpha$ with $|\alpha| \leq 2$. Here and in what follows in this proof, $C$ denotes a positive constant, depending only on $\omega, \Omega$, and the elliptic and Lipschitz constants of $A$.

Set

$$
u(t, x)=\varphi(x) v(t, x) \text { in }(0, T) \times \Omega,
$$

and denote

$$
g(t, x)=-(2\langle A(x) \nabla v(t, x), \nabla \varphi(x)\rangle+v(t, x) \operatorname{div}(A(x) \nabla \varphi(x))) \text { in }(0, T) \times \Omega,
$$

where $\langle\cdot, \cdot\rangle$ denotes the standard scalar product in $\mathbb{R}^{d}$.

We derive from (3.1) and the symmetry of $A$ that

$$
\left\{\begin{array}{cl}
\partial_{t} u-\operatorname{div}(A \nabla u)=g & \text { in }(0, T) \times \Omega, \\
u=0 & \text { on }(0, T) \times \partial \Omega .
\end{array}\right.
$$

Multiplying the equation of $u$ by $u$ and integrating by parts in $(t, T) \times \Omega$, we obtain, for $0 \leq t \leq T$,

$$
\begin{aligned}
\frac{1}{2} \int_{\Omega}|u(T, x)|^{2} d x+\int_{t}^{T} \int_{\Omega}\langle A(x) \nabla u(s, x) & , \nabla u(s, x)\rangle d x d s \\
& =\frac{1}{2} \int_{\Omega}|u(t, x)|^{2} d x+\int_{t}^{T} \int_{\Omega} g(s, x) u(s, x) d x d s .
\end{aligned}
$$


We next estimate the last term of (3.6). Since, for $x \in \Omega$ and $s \in(0, T)$,

$$
\begin{aligned}
\langle A(x) \nabla v(s, x), \nabla \varphi(x)\rangle u(s, x) & \stackrel{(3.4)}{=}\langle A(x) \varphi(x) \nabla v(s, x), \nabla \varphi(x)\rangle v(s, x) \\
& \stackrel{(3.4)}{=}\langle A(x)(\nabla u(s, x)-v(s, x) \nabla \varphi(x)), \nabla \varphi(x)\rangle v(s, x),
\end{aligned}
$$

it follows that, for $s \in(0, T)$,

$$
\left|\int_{\Omega}\langle A(x) \nabla v(s, x), \nabla \varphi(x)\rangle u(s, x) d x\right| \leq C \int_{\Omega} \delta^{-1}|\nabla u(s, x)||v(s, x)| d x+\int_{\Omega} \delta^{-2}|v(s, x)|^{2} d x .
$$

We also have, for $x \in \Omega$ and $s \in(0, T)$,

$$
|v(s, x) \operatorname{div}(A(x) \nabla \varphi(x))||u(s, x)| \leq C \delta^{-2}|v(s, x)|^{2},
$$

since $A$ is Lipschitz and $\left|\nabla_{x}^{\alpha} \varphi\right| \leq C / \delta^{|\alpha|}$ for all multi-indices $\alpha$ with $|\alpha| \leq 2$. Combining (3.7) and (3.8) yields

$$
\begin{aligned}
\left|\int_{t}^{T} \int_{\Omega} g(s, x) u(s, x) d x d s\right| \leq C \int_{t}^{T} \int_{\Omega} \delta^{-1}|\nabla u(s, x)||v(s, x)| d x d s & \\
& +\int_{t}^{T} \int_{\Omega} \delta^{-2}|v(s, x)|^{2} d x d s .
\end{aligned}
$$

Using (3.9) and the ellipticity of $A$, and applying Young's inequality, we derive from (3.6) that, for $t \in(0, T)$,

$$
\int_{\Omega}|u(T, x)|^{2} d x \leq \int_{\Omega}|u(t, x)|^{2} d x+C \delta^{-2} \int_{t}^{T} \int_{\Omega}|v(s, x)|^{2} d x d s .
$$

Integrating the above inequality with respect to $t$ from 0 to $T$, we derive that

$$
\int_{\Omega}|u(T, x)|^{2} d x \leq C \delta^{-2} T^{-1} \int_{0}^{T} \int_{\Omega}|v(s, x)|^{2} d x d s .
$$

Since $v=\varphi u, 0 \leq \varphi \leq 1, \varphi=1$ in $\omega_{\delta}$, and $\operatorname{supp} \varphi \subset \omega_{2 \delta}$, it follows that

$$
\int_{\omega_{\delta}}|v(T, x)|^{2} d x \leq C \delta^{-2} T^{-1} \int_{0}^{T} \int_{\omega_{2 \delta}}|v(t, x)|^{2} d x d t
$$

We derive from (3.3) that

$$
\int_{\omega_{\delta}}|v(T, x)|^{2} d x \leq C_{\varepsilon} \delta^{-2} T^{-1} e^{\varepsilon \sqrt{\lambda}} \int_{0}^{T} \int_{\omega}|v(t, x)|^{2} d x d t,
$$

which is the conclusion. The proof is complete.

We are ready to give

Proof of Theorem 1.1. Fix $\lambda=c_{0} / T^{2}$ where $c_{0}$ is a large positive constant determined later. Set

$$
H:=\left\{\sum_{\lambda_{i} \leq \lambda} a_{i} e^{-\lambda_{i}(T / 3-t)} e_{i}(x) ; a_{i} \in \mathbb{R}, x \in \Omega, t \in(0, T / 3)\right\} \subset L^{2}((0, T / 3) \times \Omega) .
$$

Equip $H$ with the standard scalar product in $L^{2}((0, T / 3) \times \Omega)$. Then, $H$ is a Hilbert space (of finite dimensions). 
Let $\varphi \in H$, and set

$$
v(t, x)=\varphi(T / 3-t, x) \text { for }(t, x) \in(0, T / 3) \times \Omega .
$$

It follows from the definition of $H$ in (3.10) that

$$
\left\{\begin{array}{cl}
\partial_{t} v-\operatorname{div}(A \nabla v)=0 & \text { in }(0, T / 3) \times \Omega, \\
v=0 & \text { on }(0, T / 3) \times \partial \Omega,
\end{array}\right.
$$

and moreover, $v(t=0, \cdot) \in E_{\leq \lambda}$.

By Lemma 3.1, there exist two constants $\delta \in(0,1)$ and $C_{\varepsilon}>0$, depending only on $\varepsilon, \omega, \Omega, c_{0}$, and the Lipschitz and elliptic constants of $A$, such that

$$
\|v(T / 3, \cdot)\|_{L^{2}\left(\omega_{\delta}\right)} \leq C_{\varepsilon} \delta^{-1} T^{-1 / 2}\|v\|_{L^{2}((0, T / 3) \times \Omega)} .
$$

This implies, by (3.11),

$$
\|\varphi(0, \cdot)\|_{L^{2}\left(\omega_{\delta}\right)} \leq C_{\varepsilon} \delta^{-1} T^{-1 / 2} e^{\varepsilon / T}\|\varphi\|_{L^{2}((0, T / 3) \times \omega)} \cdot
$$

Fix such constants $\delta$ and $C_{\varepsilon}$.

Fix $u_{0} \in L^{2}(\Omega)$ with $\operatorname{supp} u_{0} \subset \omega_{\delta}$. We will construct a control with support in $(0, T) \times \omega$, which steers $u_{0}$ from time 0 to 0 at time $T$ for which the cost is bounded by $C_{\varepsilon} e^{\varepsilon / T}\left\|u_{0}\right\|_{L^{2}(\Omega)}$.

Since $u_{0} \in L^{2}(\Omega)$ with supp $u_{0} \subset \omega_{\delta}$, using the Riesz representation theorem, we derive from (3.12) that there exists $f_{1} \in H$, such that

$$
\int_{\Omega} u_{0}(x) \varphi(0, x) d x=\int_{0}^{T / 3} \int_{\omega} f_{1}(s, x) \varphi(s, x) d x d s \text { for } \varphi \in H,
$$

and

$$
\left\|f_{1}\right\|_{L^{2}((0, T / 3) \times \omega)} \leq C_{\varepsilon} \delta^{-1} T^{-1 / 2} e^{\varepsilon / T}\left\|u_{0}\right\|_{L^{2}(\Omega)} .
$$

Let $u_{1} \in L^{2}\left((0, T / 3) ; H_{0}^{1}(\Omega)\right) \cap C\left([0, T / 3] ; L^{2}(\Omega)\right)$ be the unique solution of the system

$$
\left\{\begin{array}{cl}
\partial_{t} u_{1}-\operatorname{div}\left(A(x) \nabla u_{1}\right)=f_{1} \mathbb{1}_{\omega} & \text { in }(0, T / 3) \times \Omega, \\
u_{1}=0 & \text { on }(0, T / 3) \times \partial \Omega, \\
u_{1}(t=0, \cdot)=u_{0} & \text { in } \Omega .
\end{array}\right.
$$

Since

$$
\left\{\begin{array}{c}
\partial_{t} \varphi+\operatorname{div}(A \nabla \varphi)=0 \text { in }(0, T / 3) \times \Omega, \\
\varphi=0 \text { on }(0, T / 3) \times \partial \Omega,
\end{array} \quad \text { for } \varphi \in H,\right.
$$

multiplying the equation of $u_{1}$ by $\varphi(\in H)$ and integrating by parts in $(0, T / 2) \times \Omega$, we obtain

$$
\int_{\Omega} u_{1}(T / 3, x) \varphi(T / 3, x) d x-\int_{\Omega} u_{1}(0, x) \varphi(0, x) d x=\int_{0}^{T / 3} \int_{\omega} f_{1}(s, x) \varphi(s, x) d x d s \text { for } \varphi \in H .
$$

Using (3.13), we derive that

$$
\int_{\Omega} u_{1}(T / 3, x) \varphi(T / 3, x) d x=0 \text { for } \varphi \in H
$$


In other words, the projection of $u(T / 3, \cdot)$ into $E_{\leq \lambda}$ is 0 . Thus,

$$
u_{1}(T / 3, x)=\sum_{\lambda_{i}>\lambda}\left\langle u_{1}(T / 3, \cdot), e_{i}\right\rangle_{L^{2}(\Omega)} e_{i}(x) \text { in } \Omega,
$$

where $\langle\cdot, \cdot\rangle_{L^{2}(\Omega)}$ denotes the standard scalar product in $L^{2}(\Omega)$.

On the other hand, by the standard energy estimate, we have

$$
\int_{\Omega}\left|u_{1}(T / 3, x)\right|^{2} d x \leq 2 \int_{\Omega}\left|u_{1}(0, x)\right|^{2} d x+C \int_{0}^{T / 3} \int_{\omega}\left|f_{1}(s, x)\right|^{2} d s d x .
$$

We derive from (3.14) that

$$
\left\|u_{1}(T / 3, \cdot)\right\|_{L^{2}(\Omega)} \leq C_{\varepsilon} \delta^{-1} T^{-1 / 2} e^{\varepsilon / T}\left\|u_{0}\right\|_{L^{2}(\Omega)} .
$$

Let $u_{2} \in L^{2}\left((T / 3,2 T / 3) ; H_{0}^{1}(\Omega)\right) \cap C\left([T / 3,2 T / 3] ; L^{2}(\Omega)\right)$ be the unique solution of the system

$$
\left\{\begin{array}{cl}
\partial_{t} u_{2}-\operatorname{div}\left(A(x) \nabla u_{2}\right)=0 & \text { in }(0, T / 3) \times \Omega, \\
u_{2}=0 & \text { on }(0, T / 3) \times \partial \Omega, \\
u_{2}(t=T / 3, \cdot)=u_{1}(T / 3, \cdot) & \text { in } \Omega .
\end{array}\right.
$$

It follows from (3.17) and (3.18) that

$$
\left\|u_{2}(2 T / 3, \cdot)\right\|_{L^{2}(\Omega)} \leq e^{-\lambda T / 3}\left\|u_{2}(T / 3, \cdot)\right\|_{L^{2}(\Omega)} \leq C_{\varepsilon} \delta^{-1} T^{-1 / 2} e^{\varepsilon / T-\lambda T / 3}\left\|u_{0}\right\|_{L^{2}(\Omega)},
$$

which yields, since $\lambda=c_{0} / T^{2}$,

$$
\left\|u_{2}(2 T / 3, \cdot)\right\|_{L^{2}(\Omega)} \leq C_{\varepsilon} \delta^{-1} T^{-1 / 2} e^{\varepsilon / T-c_{0} /(3 T)}\left\|u_{0}\right\|_{L^{2}(\Omega)} .
$$

On the other hand, there exists $f_{3} \in L^{2}((2 T / 3, T) \times \Omega)$ with support in $[2 T / 3, T] \times \omega$, such that

$$
\left\|f_{3}\right\|_{L^{2}((2 T / 3, T) \times \Omega)} \leq C e^{C / T}\left\|u_{2}(2 T / 3, \cdot)\right\|_{L^{2}(\Omega)},
$$

and

$$
u_{3}(T, \cdot)=0 \text { in } \Omega,
$$

where $u_{3} \in L^{2}\left((2 T / 3, T) ; H_{0}^{1}(\Omega)\right) \cap C\left([2 T / 3, T] ; L^{2}(\Omega)\right)$ is the unique solution of the system

$$
\left\{\begin{array}{cl}
\partial_{t} u_{3}-\operatorname{div}\left(A(x) \nabla u_{3}\right)=f_{3} & \text { in }(2 T / 3, T) \times \Omega, \\
u_{3}=0 & \text { on }(T / 2, T) \times \partial \Omega, \\
u_{3}(t=2 T / 3, \cdot)=u_{2}(2 T / 3, \cdot) & \text { in } \Omega .
\end{array}\right.
$$

Define $f \in L^{2}((0, T) \times \Omega)$ as follows

$$
f(t, x)=\left\{\begin{array}{cl}
f_{1} \mathbb{1}_{\omega} & \text { in }(0, T / 3) \times \Omega, \\
0 & \text { in }(T / 3,2 T / 3) \times \Omega, \\
f_{3} & \text { in }(2 T / 3, T) \times \Omega .
\end{array}\right.
$$

Since supp $f_{3} \subset[2 T / 3, T] \times \omega$, it follows that

$$
\operatorname{supp} f \subset[0, T] \times \bar{\omega} .
$$


Let $u \in L^{2}\left((0, T) ; H_{0}^{1}(\Omega)\right) \cap C\left([0, T] ; L^{2}(\Omega)\right)$ be the unique solution of the system

$$
\left\{\begin{array}{cl}
\partial_{t} u-\operatorname{div}(A(x) \nabla u)=f & \text { in }(0, T) \times \Omega, \\
u=0 & \text { on }(T / 2, T) \times \partial \Omega, \\
u(t=0, \cdot)=u_{0} & \text { in } \Omega .
\end{array}\right.
$$

It follows from (3.15), (3.19), (3.22), and (3.23) that

$$
u_{3}(T, \cdot)=0 \text { in } \Omega .
$$

Combining (3.14) and (3.21), and using (3.20), we deduce that

$$
\|f\|_{L^{2}((0, T) \times \Omega)} \leq C_{\varepsilon} \delta^{-1} T^{-1 / 2} e^{\varepsilon / T}\left\|u_{0}\right\|_{L^{2}(\Omega)}\left(1+e^{-c_{0} /(3 T)+C / T}\right) .
$$

By fixing $c_{0}$ such that $c_{0} / 3 \geq C$, we obtain

$$
\|f\|_{L^{2}((0, T) \times \Omega)} \leq C_{\varepsilon} \delta^{-1} T^{-1 / 2} e^{\varepsilon / T}\left\|u_{0}\right\|_{L^{2}(\Omega)} .
$$

The conclusion follows by replacing $\varepsilon$ by $\varepsilon / 2$ and noting that

$$
T^{-1 / 2} e^{\varepsilon /(2 T)} \leq C_{\varepsilon} e^{\varepsilon / T}
$$

this follows by considering the case $T \geq \varepsilon$ and the case $0<T<\varepsilon$.

Remark 3.2. The conclusion of Theorem 1.1 also holds if in the definition of $c(T, w, D)$, one additionally requires that supp $f \Subset[0, T] \times \omega$. The conclusion in this case follows by applying the established result for the set $\{x \in \omega ; \operatorname{dist}(x, \partial \omega) \geq \gamma\}$ for small $\gamma$ after noting that the constant $\delta$ for such a set is independent of $\gamma$ for small $\gamma$.

Acknowledgement: The author thanks Jean-Michel Coron for his interest in the problem and for many interesting discussions. The author also thanks Kim Dang Phung for discussions on the approach of Gilles Lebeau and Luc Robbiano. This work was completed during his visit to Laboratoire Jacques Louis Lions. The author thanks the laboratory for its hospitality.

\section{REFERENCES}

[1] Jean-Michel Coron and Sergio Guerrero, Singular optimal control: a linear 1-D parabolic-hyperbolic example, Asymptot. Anal. 44 (2005), no. 3-4, 237-257. MR 2176274

[2] Jérémi Dardé and Sylvain Ervedoza, On the cost of observability in small times for the one-dimensional heat equation, Anal. PDE 12 (2019), no. 6, 1455-1488. MR 3921310

[3] Sylvain Ervedoza and Enrique Zuazua, Sharp observability estimates for heat equations, Arch. Ration. Mech. Anal. 202 (2011), no. 3, 975-1017. MR 2854675

[4] Enrique Fernández-Cara and Enrique Zuazua, The cost of approximate controllability for heat equations: the linear case, Adv. Differential Equations 5 (2000), no. 4-6, 465-514. MR 1750109

[5] Andrei V. Fursikov and O. Yu. Imanuvilov, Controllability of evolution equations, Lecture Notes Series, vol. 34, Seoul National University, Research Institute of Mathematics, Global Analysis Research Center, Seoul, 1996. MR 1406566

[6] Olivier Glass, A complex-analytic approach to the problem of uniform controllability of a transport equation in the vanishing viscosity limit, J. Funct. Anal. 258 (2010), no. 3, 852-868. MR 2558179

[7] Camille Laurent and Matthieu Léautaud, Quantitative unique continuation for operators with partially analytic coefficients. Application to approximate control for waves, J. Eur. Math. Soc. (JEMS) 21 (2019), no. 4, 9571069. MR 3941459

[8] Gilles Lebeau and Luc Robbiano, Contrôle exact de l'équation de la chaleur, Comm. Partial Differential Equations 20 (1995), no. 1-2, 335-356. MR 1312710 
[9] Gilles Lebeau and Enrique Zuazua, Null-controllability of a system of linear thermoelasticity, Arch. Rational Mech. Anal. 141 (1998), no. 4, 297-329. MR 1620510

[10] Pierre Lissy, A link between the cost of fast controls for the 1-D heat equation and the uniform controllability of a 1-D transport-diffusion equation, C. R. Math. Acad. Sci. Paris 350 (2012), no. 11-12, 591-595. MR 2956149

[11] Pierre Lissy, Explicit lower bounds for the cost of fast controls for some 1-D parabolic or dispersive equations, and a new lower bound concerning the uniform controllability of the 1-D transport-diffusion equation, J. Differential Equations 259 (2015), no. 10, 5331-5352. MR 3377528

[12] Luc Miller, How violent are fast controls for Schrödinger and plate vibrations?, Arch. Ration. Mech. Anal. 172 (2004), no. 3, 429-456. MR 2062431

[13] Luc Miller, The control transmutation method and the cost of fast controls, SIAM J. Control Optim. 45 (2006), no. 2, 762-772. MR 2246098

[14] Hoai-Minh Nguyen, Asymptotic behavior of solutions to the Helmholtz equations with sign changing coefficients, Trans. Amer. Math. Soc. 367 (2015), no. 9, 6581-6595. MR 3356948

[15] Hoai-Minh Nguyen, Cloaking an arbitrary object via anomalous localized resonance: the cloak is independent of the object, SIAM J. Math. Anal. 49 (2017), no. 4, 3208-3232. MR 3689138

[16] Hoai-Minh Nguyen, Cloaking property of a plasmonic structure in doubly complementary media and threesphere inequalities with partial data, preprint, https://arxiv.org/abs/1912.09098.

[17] Louis Nirenberg, On elliptic partial differential equations, Ann. Scuola Norm. Sup. Pisa Cl. Sci. (3) 13 (1959), 115-162. MR 109940

[18] Kim Dang Phung, Observability and control of Schrödinger equations, SIAM J. Control Optim. 40 (2001), no. 1, 211-230. MR 1855313

[19] Kim Dang Phung, Waves, damped wave and observation, Some problems on nonlinear hyperbolic equations and applications, Ser. Contemp. Appl. Math. CAM, vol. 15, Higher Ed. Press, Beijing, 2010, pp. $386-412$. MR 2816460

[20] Luc Robbiano, Fonction de coût et contrôle des solutions des équations hyperboliques, Asymptotic Anal. 10 (1995), no. 2, 95-115. MR 1324385

[21] Thomas I. Seidman, How violent are fast controls?, Math. Control Signals Systems 1 (1988), no. 1, 89-95. MR 923278

[22] Gerald Tenenbaum and Marius Tucsnak, New blow-up rates for fast controls of Schrödinger and heat equations, J. Differential Equations 243 (2007), no. 1, 70-100. MR 2363470

(Hoai-Minh Nguyen) Ecole Polytechnique Fédérale de Lausanne, EPFL, CAMA, Station 8, CH-1015 LAUSANNe, SwitzerLAND.

Email address: hoai-minh.nguyen@epfl.ch 